\title{
Artificial avidin-based receptors for a panel of small molecules
}

Soili I. Lehtonen ${ }^{\dagger}$, Antti Tullila ${ }^{\ddagger}$, Nitin Agrawal ${ }^{\S \#}$, Sampo Kukkurainen ${ }^{\dagger} \pm$, Niklas Kähkönen ${ }^{\dagger}$, Masi Koskinen ${ }^{\dagger}$,

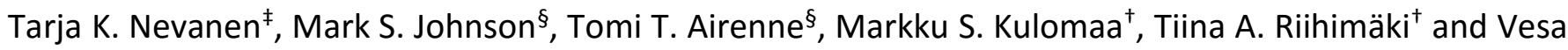
P. Hytönen ${ }^{\dagger} \pm *$

+ BioMediTech, Biokatu 6, FI-33014 University of Tampere, Tampere, Finland

‡ VTT Technical Research Centre of Finland, Tietotie 2, FI-02044 Espoo, Finland

$\S$ Structural Bioinformatics Laboratory, Biochemistry, Faculty of Science and Engineering, Åbo Akademi University, Tykistökatu 6A, FI-20520 Turku, Finland

${ }^{ \pm}$Fimlab Laboratories, Biokatu 4, FI-33520 Tampere, Finland

\# Equal contribution

* To whom correspondence should be addressed. Tel: +358 40190 1517; Fax: +358 33641 471; Email: vesa.hytonen@uta.fi

\section{Supporting Information}

Supplemental Figures 1-5; Supplemental Tables 1-4; Supplemental Results and Discussion; Supplemental Methods; Supplemental References 


\section{Supplemental Figures}

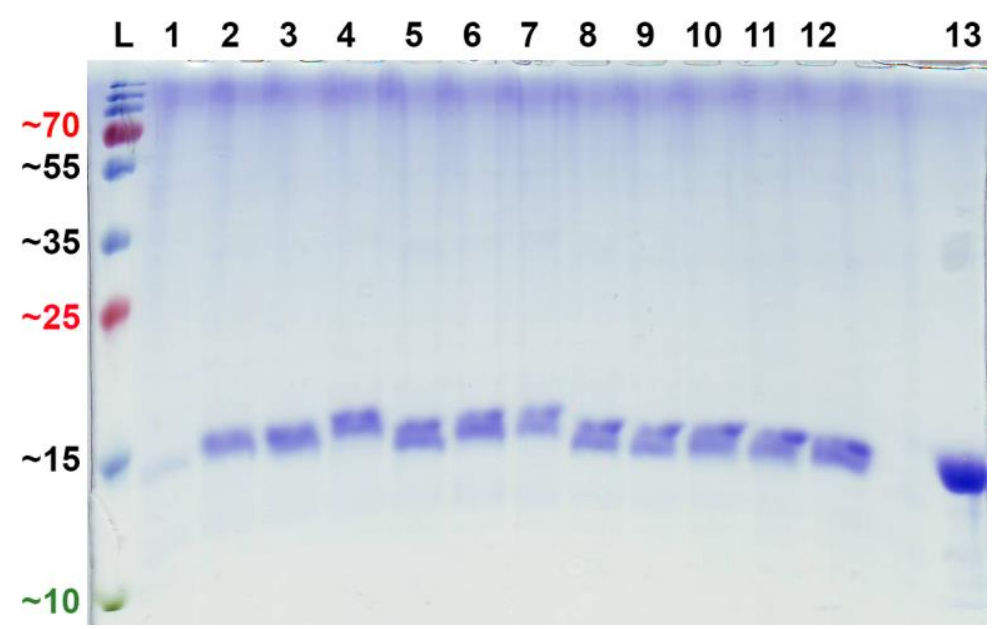

Supplementary Figure 1. Coomassie stained SDS-PAGE analysis of purified antidins. His-tagged proteins were produced in E. coli BL-21(Al) and purified by Ni-NTA affinity chromatography. Isolated proteins ( 1 $\mu \mathrm{g})$ were subjected to SDS-PAGE analysis and stained with Coomassie. 1) sbAvd-1; 2) sbAvd-2; 3) sbAvd2(I117Y); 4) sbAvd-3; 5) sbAvd-4; 6) sbAvd-5; 7) sbAvd-6; 8) hbAvd-1; 9) hbAvd-2; 10) cabAvd-1; 11) cabAvd-2; 12) fabAvd-1. As a positive control, wt avidin produced in E. coli ( 2.5 $\mu \mathrm{g})$ was used (13). 

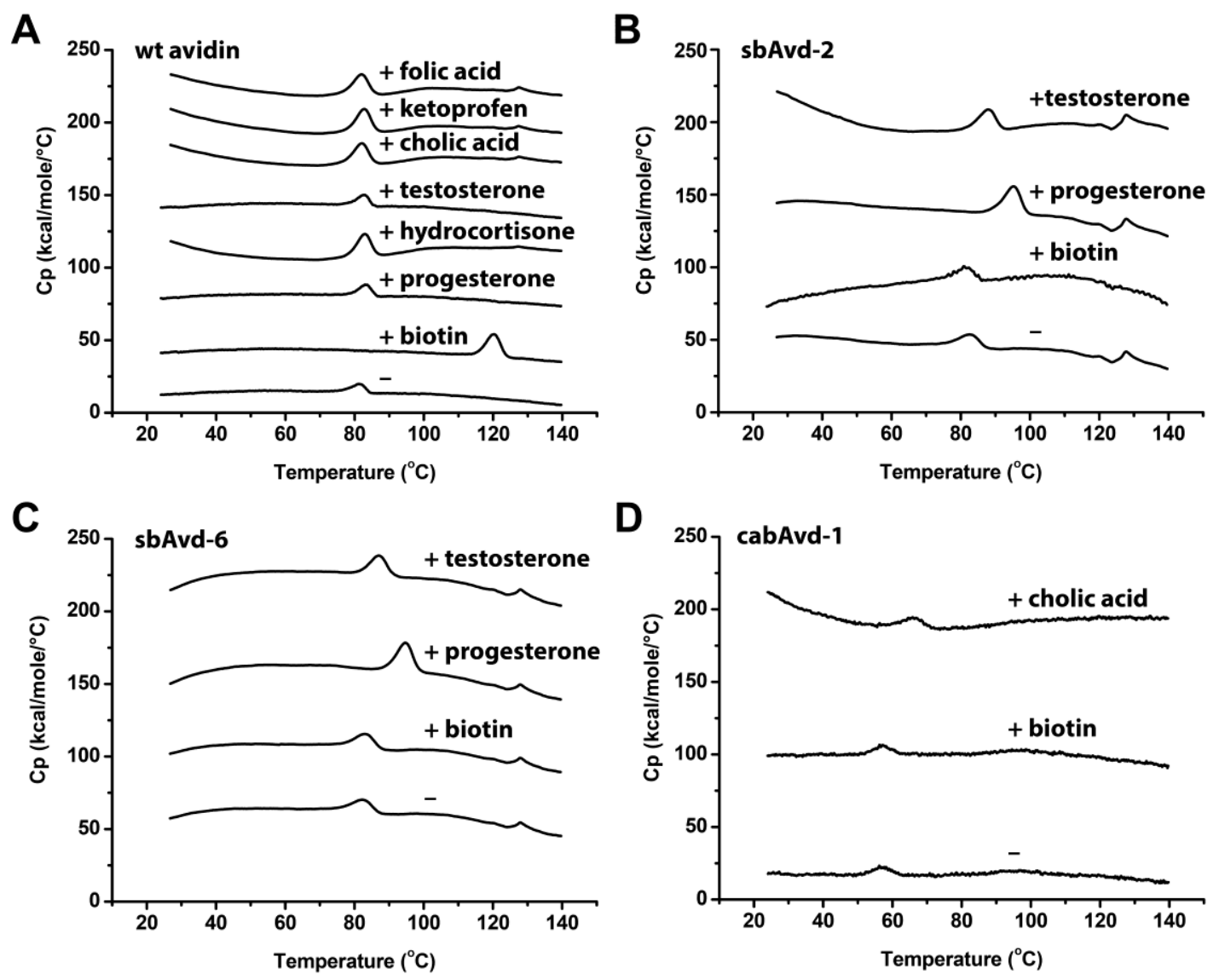

Supplementary Figure 2. DSC results of wt avidin, sbAvd-2, sbAvd-6 and cabAvd-1. The thermograms are arbitrarily displaced along the ordinate for ease of presentation. A) Thermograms of wt avidin without ligands and in the presence of ligands used in the study (biotin, progesterone, hydrocortisone, testosterone, cholic acid, ketoprofen, and folic acid). Wt avidin showed high thermal stability in DSC analysis. When biotin was added, the $\mathrm{T}_{\mathrm{m}}$ value increased by $37.9^{\circ} \mathrm{C}$ (Table 3 ), whereas other small ligands did not increase the $T_{m}$ value of wt avidin significantly. B) Thermogram of sbAvd-2 without ligand and in the presence of biotin, progesterone or testosterone. C) Thermogram of sbAvd-6 without ligand and in the presence biotin, progesterone or testostrone. In the case of B) sbAvd-2 and C) sbAvd-6, the addition of progesterone induced a significant shift to the peak $\left(13.2^{\circ} \mathrm{C}\right.$ and $12.0^{\circ} \mathrm{C}$ respectively). D) Thermogram of cabAvd-1 without ligand and in the presence of biotin or cholic acid. CabAvd-1 has the lowest biotinbinding affinity among the studied antidins. 

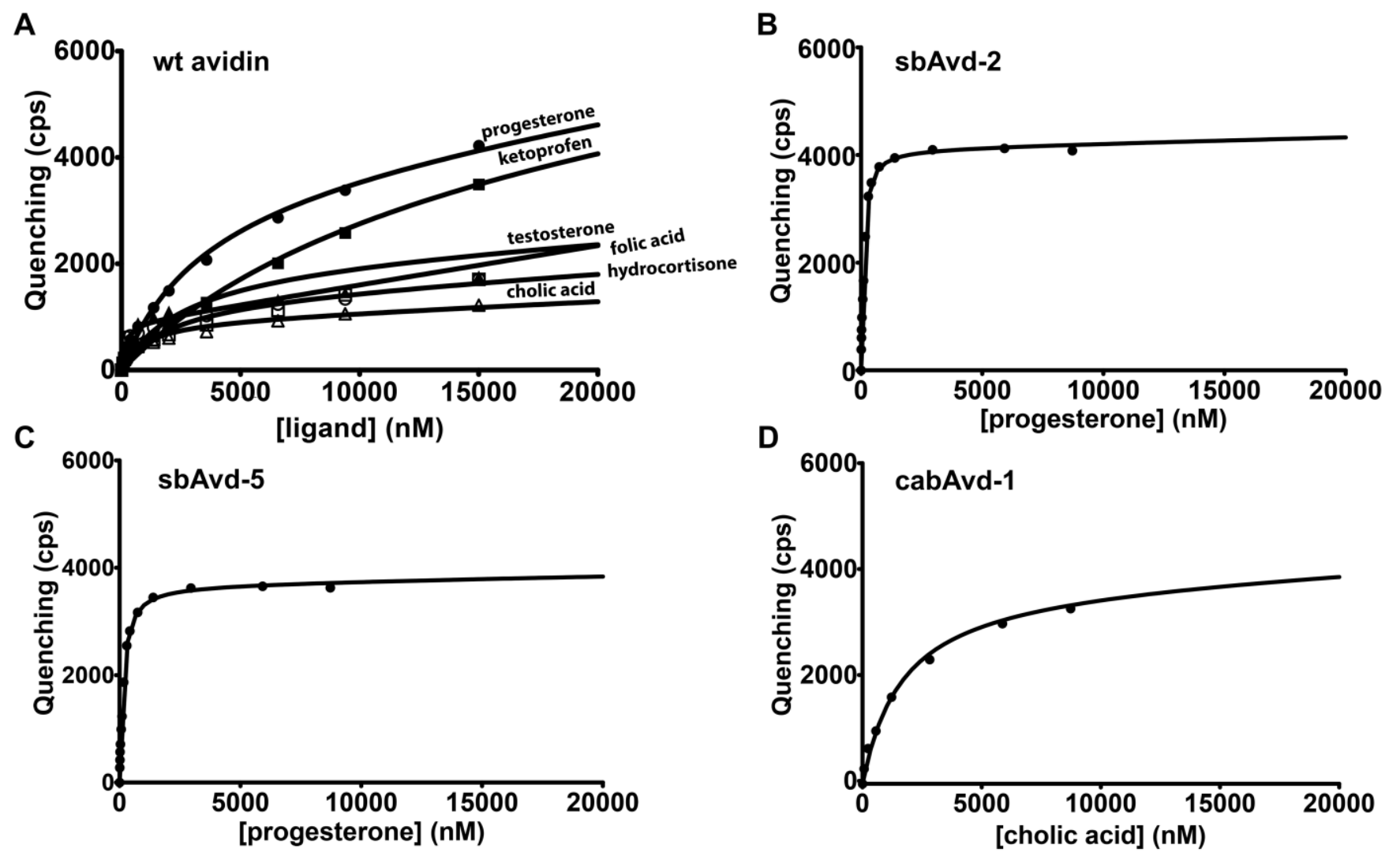

Supplementary Figure 3. Binding affinities of unconjugated molecules to antidins. The dissociation constant $\left(\mathrm{K}_{\mathrm{d}}\right)$ was determined by utilizing the intrinsic fluorescence originating from the aromatic amino acid residues of avidin and the fluorescence quenching caused by ligand binding. A) The fluorescence quenching of wt avidin caused by the binding of the small molecules (progesterone, hydrocortisone, testosterone, cholic acid, ketoprofen or folic acid) was negligible except in the case of progesterone ( $K_{d}$ $\sim 3 \mu \mathrm{M}$, Table 3). In comparison, progesterone showed strong binding to sbAvds; as an example, sbAvd-2 (B) and sbAvd-5 (C) are shown. The determined $K_{d}$ values for these proteins with progesterone are 111 $\mathrm{nM}$ and $108 \mathrm{nM}$, respectively (Table 3). The binding affinity $\left(\mathrm{K}_{\mathrm{d}}\right)$ of cabAvd-1 (D) towards cholic acid was 1.7 $\mu \mathrm{M}$ (Table 3). Cps, counts per second. 
A

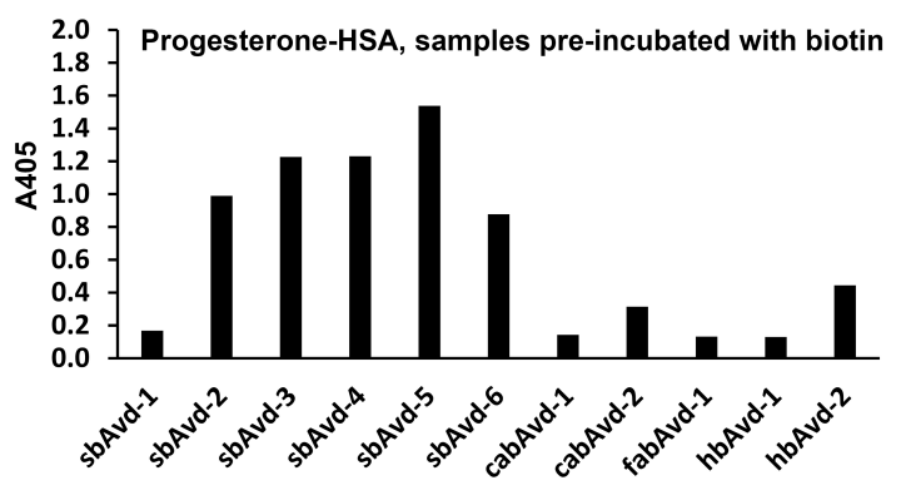

B

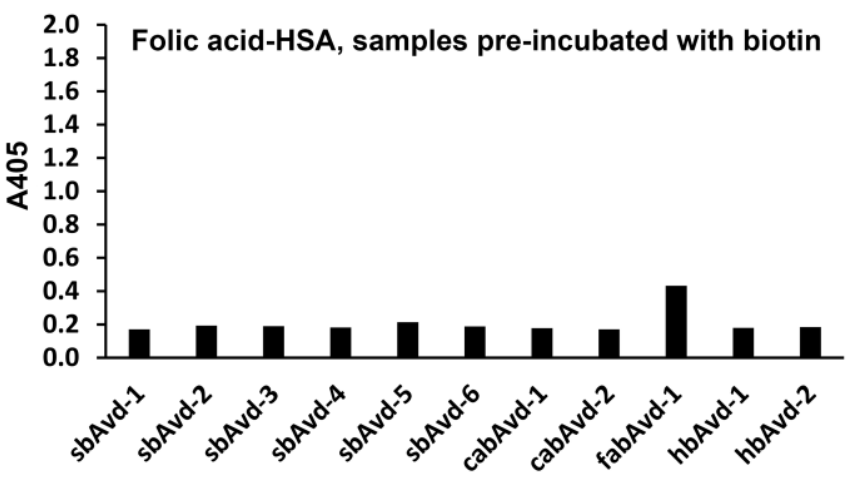

Supplementary Figure 4. Biotin-inhibition microplate assay. Small molecule ligand binding of antidins was competed with pre-incubated biotin. In the assay, a protein concentration of $\sim 1 \mu \mathrm{gl}^{-1}$ was used and the protein sample was pre-incubated with $10 \mu \mathrm{M}$ D-biotin (Biochemica, Fluka, 14400) as previously described ${ }^{22}$. The binding of antidins pre-incubated with biotin was studied on A) progesterone-HSA coated wells and B) folic acid-HSA coated wells. When the results are compared with Figure $2 \mathrm{~A}$ and $2 \mathrm{~F}$, it is clear, that the ligand binding preference of sbAvd-2-6 is significantly altered. 


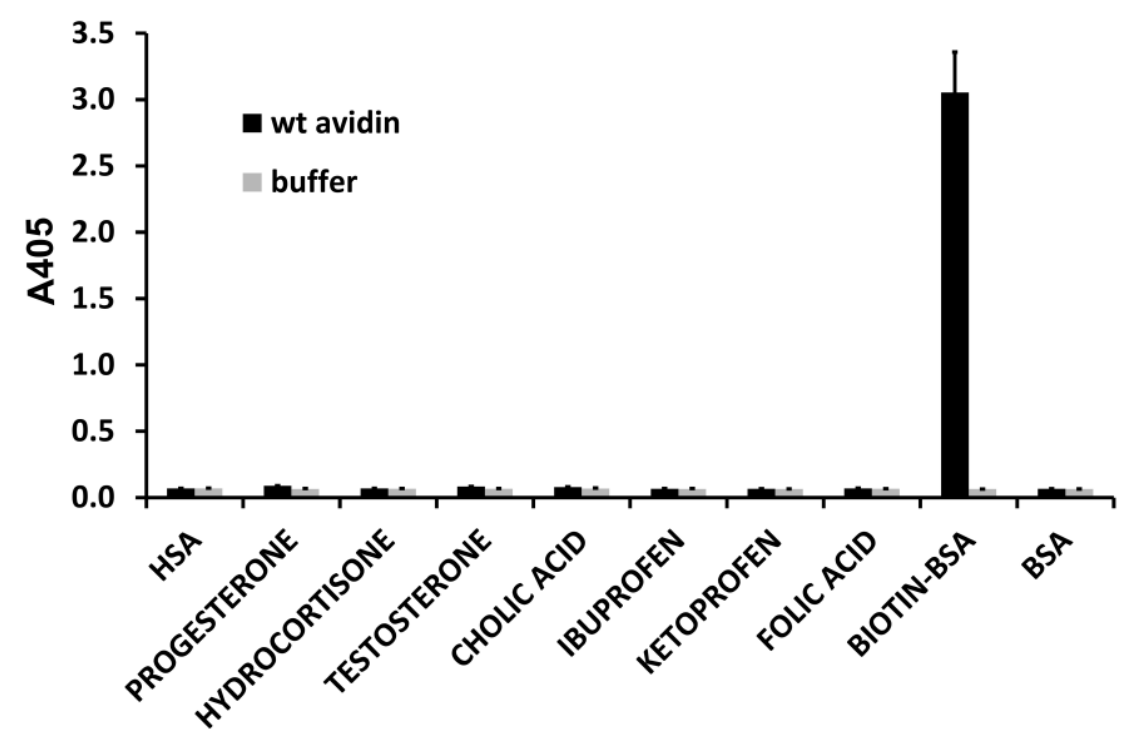

Supplementary Figure 5. Ligand binding of small molecule to wt avidin. In the microplate assay, HSAconjugated ligands (progesterone, hydrocortisone, testosterone, cholic acid, ibuprofen, ketoprofen and folic acid) were used. Wt avidin showed responses similar to the buffer, which was used as a negative control. As a positive control, BSA-conjugated biotin was used. 


\section{Supplemental Tables}

Supplementary Table 1. Gel filtration chromatography with SEC-SLS analysis

\begin{tabular}{lcccc}
\hline protein sample & elution volume & $\begin{array}{c}\text { molecular weight } \\
\text { (kDa) }\end{array}$ & $\begin{array}{c}\text { hydrodynamic radius } \\
\text { (nm) }\end{array}$ \\
& SEC (ml) & SEC-SLS & theoretical & SEC-DLS \pm SD a \\
\hline BSA & 1.84 & 65.5 & 66.4 & $4.07 \pm 0.19$ \\
sbAvd-1 & 2.05 & 61.0 & 62.6 & $3.57 \pm 0.22$ \\
sbAvd-2 & 2.05 & 64.1 & 62.7 & $3.66 \pm 0.18$ \\
sbAvd-2(I117Y) & 2.07 & 62.7 & 62.9 & $3.57 \pm 0.21$ \\
sbAvd-2(N118D) & $\mathrm{n} / \mathrm{d}$ b & & & \\
sbAvd-3 & 2.06 & 57.5 & 62.7 & $3.57 \pm 0.26$ \\
sbAvd-4 & 2.20 & 57.9 & 63.0 & $\mathrm{n} / \mathrm{a}$ \\
sbAvd-5 & 2.10 & 55.4 & 62.9 & $3.46 \pm 0.20$ \\
sbAvd-6 & 2.06 & 60.6 & 62.7 & $3.70 \pm 0.51$ \\
cabAvd-1 & 2.06 & 46.5 & 62.1 & $3.12 \pm 0.25$ \\
cabAvd-2 & 2.05 & 49.4 & 61.7 & $3.54 \pm 0.20$ \\
cabAvd-2(I117Y) & $\mathrm{n} / \mathrm{d}$ & & & \\
hbAvd-1 & 2.03 & 55.0 & 62.0 & $3.71 \pm 0.21$ \\
hbAvd-2 & 2.14 & 65.4 & 61.8 & $3.53 \pm 0.28$ \\
fabAvd-1 & 1.99 & 54.8 & 61.9 & $3.67 \pm 0.18$ \\
fabAvd-1(I117Y) & $\mathrm{n} / \mathrm{d}$ & & & $3.32 \pm 0.30$ \\
bwtAvd & 2.00 & 51.8 & 58.7 & \\
\hline SD, Standard deviation & & & & \\
\hline
\end{tabular}

a SD, Standard deviation

${ }^{\mathrm{b}} \mathrm{n} / \mathrm{d}$, not determined with SEC-SLS analysis. However, the preliminary gel filtration analysis with ÄKTA Purifier and Superdex 200 10/300 GL column (GE healthcare) showed that this mutant, as well as the other mutants, was tetrameric.

${ }^{\mathrm{c}} \mathrm{n} / \mathrm{a}$, DLS signal too low to obtain values 
Supplementary Table 2. Panning conditions

\begin{tabular}{|c|c|c|c|c|c|c|}
\hline Round & Phages & Depletion & Beads & Incubation & PBST washes & Elution \\
\hline $1 s t$ & $\begin{array}{l}200 \mu \mathrm{l} \mathrm{PEG} \\
\text { precipitated phages } \\
+200 \mu \mathrm{I} \text { PBST }(0,05 \%)\end{array}$ & $20 \mu \mathrm{g} \mathrm{AP}{ }^{a}$ & $5 \mu l$ & overnight & $5 \times 20 s$ & $\begin{array}{c}20 \text { min in glycine- } \mathrm{HCl} \\
(\mathrm{pH} \mathrm{2.2)}\end{array}$ \\
\hline 2nd & $\begin{array}{l}200 \mu \mathrm{l} \text { of } \\
\text { supernatant from } \\
\text { the phage cultures }{ }^{b}\end{array}$ & $20 \mu \mathrm{g} A P$ & $4 \mu l$ & $4 \times 15 \min$ & $4 \times 20 s$ & $\begin{array}{c}20 \mathrm{~min} \text { in glycine- } \mathrm{HCl} \\
(\mathrm{pH} \mathrm{2.2)}\end{array}$ \\
\hline $3 r d$ & $\begin{array}{l}200 \mu l \text { of } \\
\text { supernatant from } \\
\text { the phage cultures }{ }^{b}\end{array}$ & $20 \mu \mathrm{g} A P$ & $3 \mu l$ & $4 \times 15 \min$ & $4 \times 20 s$ & $\begin{array}{c}20 \text { min in glycine- } \mathrm{HCl} \\
(\mathrm{pH} \mathrm{2.2)}\end{array}$ \\
\hline 4th & $\begin{array}{l}200 \mu l \text { of } \\
\text { supernatant from } \\
\text { the phage cultures }{ }^{b}\end{array}$ & $20 \mu \mathrm{g} A P$ & $2 \mu l$ & $2 \times 15 \min$ & $4 \times 1 \mathrm{~min}$ & $\begin{array}{c}20 \text { min in glycine- } \mathrm{HCl} \\
(\mathrm{pH} \mathrm{2.2)}\end{array}$ \\
\hline 5th & $\begin{array}{l}200 \mu l \text { of } \\
\text { supernatant from } \\
\text { the phage cultures }^{b}\end{array}$ & $20 \mu \mathrm{g} A P$ & $1 \mu l$ & $2 \times 7.5 \mathrm{~min}$ & $4 \times 2 \min$ & $\begin{array}{c}20 \text { min in glycine- } \mathrm{HCl} \\
(\mathrm{pH} \mathrm{2.2)}\end{array}$ \\
\hline
\end{tabular}

${ }^{a}$ alkaline phosphatase (AP)

${ }^{b}$ incubation was performed in the presence of Tween (0.05\%) 
Supplementary Table 3. Efficiency of selection. The output/input ratios of the phage titers.

\begin{tabular}{|c|c|c|c|c|c|}
\hline Target & $\begin{array}{c}1^{\text {st }} \\
\left(\times 10^{-3}\right) \\
\end{array}$ & $\begin{array}{c}2^{\text {nd }} \\
\left(\times 10^{-3}\right)\end{array}$ & $\begin{array}{c}\text { 3rd } \\
\left(\times 10^{-3}\right)\end{array}$ & $\begin{array}{c}4 \text { th } \\
\left(\times 10^{-3}\right)\end{array}$ & $\begin{array}{c}5 \text { th } \\
\left(\times 10^{-3}\right)\end{array}$ \\
\hline Progesterone & 0.693 & 8.584 & 31.220 & 88.629 & 110.860 \\
\hline Hydrocortisone & 0.648 & 6.127 & 116.058 & 167.266 & 12.644 \\
\hline Testosterone & 0.490 & 0.632 & 41.071 & 245.342 & 30.435 \\
\hline Cholic acid & 0.030 & 2.319 & 90.278 & 47.183 & 12.195 \\
\hline Ibuprofen & 0.004 & 0.064 & 0.081 & 4.000 & 2.143 \\
\hline Ketoprofen & 0.723 & 9.355 & 76.800 & 200.450 & 234.653 \\
\hline Folic acid & 0.182 & 4.703 & 96.947 & 87.898 & 5.773 \\
\hline Control selection & 1.348 & 6.538 & 35.821 & 74.297 & 12.335 \\
\hline
\end{tabular}


Supplementary Table 4. A list of primers used.

\begin{tabular}{|c|c|c|}
\hline primer & sequence 5'-3' & used for \\
\hline 3_4R_1_3' & CTCTTTGATCTCATTTGARNNRNNRNNRNNRNNRNNGATGTAGGTGCCTGTGAATTC & construction of the AvLib-3 ${ }^{a}$ \\
\hline 3_4_loop_5' & TCAAATGAGATCAAAGAG & construction of the AvLib-3 ${ }^{a}$ \\
\hline 1_2R_2_5' & AAC ATG ACC ATC GGG GCT & construction of the AvLib-2 \\
\hline 1_2R_1_3' & AGC CCC GAT GGT CAT GTT RNN RNN RNN RNN RNN GGT CCA TTT CCC AGT CAG & construction of the AvLib-2 \\
\hline 5_6R_2_5' & ACC ACT GTC TTC ACG GGC & construction of the AvLib-1 and AvLib-2 \\
\hline 5_6R_1_3' & GCC CGT GAA GAC AGT GGT NNN NNN NNN NNN CTT CCA ATT GAC GGT GAA & construction of the AvLib-1 \\
\hline 5_6R_1_RNN_3' & GCC CGT GAA GAC AGT GGT RNN RNN RNN RNN CTT CCA ATT GAC GGT GAA & construction of the AvLib-2 \\
\hline Avd_Nhel_5' & TAT TGC TAG CTG CAC AAC CAG CAA TGG CAG CCA GAA AGT GCT CGC TGA C & construction of the AvLib-1-3 ${ }^{a}$ \\
\hline Uusi_Notl_3' & AAC CGT ATT TGC GGC CGC CTC CTT CTG TGT GCG CAG GC & construction of the AvLib-1-3 ${ }^{a}$ \\
\hline 1867 & ACTCATTAGGCACCCCAGGC & DNA sequencing \\
\hline 886 & GCTCAAGTCGGTGACGGTGATAAT & DNA sequencing \\
\hline plII_Rev2_RR & CGGTCATAGCCCCCTTATTAGC & DNA sequencing \\
\hline Chim_to_1_5' & GCCGCCGTTACGGCCTCTGGTGTTGCCTCGGCTCAGACCGTGGCCAGAAAGTGCTCGCTG & addition of half of the OmpA-signal peptide \\
\hline Chim_to_2_5' & CACCATGAACAAACCCTCCAAATTCGCTCTGGCGCTTGCCTTCGCCGCCGTTACGGCCTC & $\begin{array}{l}\text { addition of the rest of the OmpA-signal peptide } \\
\text { (suitable for TOPO cloning) }\end{array}$ \\
\hline Avi_phage_histag_stop_3' & TCAATGATGGTGGTGATGATGCTCCTTCTGTGTGCGCAGGCGAGTGAAGATGTT & addition of the $6 x$ His-tag \\
\hline I117Y_1 & CAGGGTCGGCTACAACATCTTC & QuikChange-mutagenesis to introduce I117Y \\
\hline I117Y_2 & GAAGATGTTGTAGCCGACCCTG & QuikChange-mutagenesis to introduce I117Y \\
\hline N118D_1 & GCTACCAGGGTCGGCATCGATATCTTCACTCGCC & QuikChange-mutagenesis to introduce N118D \\
\hline N118D_2 & GGCGAGTGAAGATATCGATGCCGACCCTGGTAGC & QuikChange-mutagenesis to introduce N118D \\
\hline
\end{tabular}

${ }^{a}$ AvLib-3 referred to sbAvd-1 L3,4 library in ${ }^{22}$ 


\section{Supplemental Results and Discussion}

\section{Library design and selection}

Different carrier proteins, AP and HSA, were used for the selection and screening, respectively, in order to reduce the amount of false positives resulting from phages binding to carrier molecules. Up to 96 clones from the fifth panning round master plates were DNA sequenced, and most of the sequences had the correct reading frame (the sequences were intact and in frame with the C-terminal plII). In all cases, enriched sequences were obtained (Table 2). The enriched sequences from the progesterone, testosterone and ketoprofen selections were from the AvLib-3 library, whereas the other ligands enriched forms from the AvLib-2 library.

\section{Thermal stability of sbAvds}

The DSC measurements showed that all of sbAvds had retained the high thermal stability of wt avidin with $\mathrm{T}_{\mathrm{m}}$ values around $80^{\circ} \mathrm{C}$ (Table 3, Supplementary Figure 2 ), indicating that the modifications did not have a destabilizing effect on these protein scaffolds. Addition of progesterone increased the $T_{m}$ values by $10.1-13.2^{\circ} \mathrm{C}$ in all sbAvds (Table 3 ), whereas the addition of testosterone had a smaller effect on the $T_{m}$ values $\left(\Delta T_{m}=2.5-5.6^{\circ} \mathrm{C}\right)$. For sbAvd-5 and sbAvd-6, the change in the $T_{m}$ values was also analyzed in the presence of ketoprofen, which had only a mild effect on the stability of the proteins $\left(\Delta \mathrm{T}_{\mathrm{m}}=1.2-1.5^{\circ} \mathrm{C}\right.$, Table 3$)$. In DSC analysis, the addition of biotin had only a minor effect on the $T_{m}$ values of the mutants as compared to those of the steroids (Table 3 ). The $\Delta T_{m}$ values of sbAvds in the presence of different ligands correlated well with their $K_{d}$ values: The bigger the difference, the higher the affinity. 


\section{Control measurements of wt avidin with the small ligands}

The interaction of wt avidin with the small ligands used in this study was analyzed with various methods (DSC, microplate assay and intrinsic fluorescence). In general, only a low-level response was seen, indicating that wt avidin had only negligible affinities towards the ligands. The $T_{m}$ value of avidin determined by DSC increased by $37.9^{\circ} \mathrm{C}$ when biotin was added, whereas in the case of other ligands the stabilizing effect was only $0.6-2.2^{\circ} \mathrm{C}$ (Table 3, Supplementary Figure $2 \mathrm{~A}$ ). In the microplate assay, wt avidin showed responses for the HSA-conjugated ligands similar to the buffer used as a negative control (Supplementary Figure 5). Additionally, the affinity of wt avidin to the ligands studied here was analyzed by measuring the changes in intrinsic fluorescence, which showed negligible changes (Supplementary Figure 3A), except in the case of progesterone ( $K_{d} \sim 3 \mu M$, Table 3).

\section{Crystal structure of sbAvd-2(I117Y)}

There were several unidentified blobs of density in the electron density maps that were clearly not representing protein and were larger than those typically observed for water molecules. Ten of these blobs of electron density were modeled as chloride ions based on their shape and size and the fact that the well solution used for crystallization had sodium chloride. However, these blobs of density may represent some other ions or small molecules, too. Two electron density blobs within the ligandbinding pocket were not modeled.

The electron density around His16 of sbAvd-2(I117Y) is well defined even though the neighboring residues, Arg13, Met14 and Asn15 of sbAvd-2(I117Y), which are mainly exposed to solvent, have high B-factors, and thus have more error in their coordinates. 


\section{Supplemental Methods}

Preparation of the libraries for phage display. Three avidin mutant libraries (AvLib-1-3 libraries, see Table 1) with randomization targeted to loop regions of the $\beta$-barrel structure were prepared using a two-step PCR strategy as previously described ${ }^{22}$ (primers are listed in Supplementary Table 4). PCR fragments of correct size were ligated into a phagemid vector (pBluescript SK+, VTT Technical Research Centre of Finland) ${ }^{22}$ and the resulting plasmids were electroporated into E. coli XL1-Blue (Stratagene). AvLib-1-3 phage display libraries were constructed separately: Ten parallel electroporation reactions were prepared from each library using $0.2 \mu \mathrm{g}(1 \mu \mathrm{l})$ DNA per $40 \mu \mathrm{l}$ of XL1-Blue (Stratagene). After a onehour incubation, a $50 \mu \mathrm{l}$ sample of each transformation was plated onto LBamp plates (100 $\mu \mathrm{g} \mathrm{ml} \mathrm{m}^{-1}$ ampicillin and $0.1 \%$ glucose). The remaining transformations of the same library were pooled together and diluted with SB medium containing ampicillin $\left(100 \mu \mathrm{g} \mathrm{ml} \mathrm{m}^{-1}\right)$ and tetracycline $\left(10 \mu \mathrm{g} \mathrm{m} \mathrm{m}^{-1}\right)$. Consecutive steps to produce and purify phages are described in the article.

Conjugation reactions. Ligands (progesterone 3-(O-carboxymethyl)oxime; hydrocortisone 3-(Ocarboxymethyl)oxime; testosterone 3-(O-carboxymethyl)oxime; cholic acid; S-(+)-ibuprofen; S-(+)ketoprofen; folic acid (Sigma-Aldrich) were conjugated to the carrier proteins (alkaline phosphatase (AP) (Sigma-Aldrich) or human serum albumin (HSA) (Sigma-Aldrich) covalently using carbodiimide/Nhydroxysuccinimide (EDC/NHS) -crosslinker chemistry (Thermo Scientific). Conjugated small moleculeprotein complexes were then purified from excess of conjugation reagents using size exclusion chromatography and success of conjugation was verified with MALDI-TOF mass spectrometry (Bruker).

Coating and blocking of the magnetic beads. Magnetic beads (Dynabeads ${ }^{\circledR}$ M-270 Epoxy, Invitrogen) were coated with AP-conjugated ligands according to instructions from the manufacturer. The coating of the beads was then confirmed by Micro BCA Protein Assay Kit (Thermo Scientific) essentially as 
described ${ }^{32}$ using $10 \mu$ of coated beads and the HSA standard curve. The coated beads were blocked with $2.5 \%$ skimmed milk-PBS in a shaker for $2.5 \mathrm{~h}$, washed four times and finally re-suspended in PBS $(\mathrm{pH} 7.4)$.

Semi-automated biopanning procedure. Five rounds of semi-automated biopanning on microplates ${ }^{32}$ were performed with a KingFisher ${ }^{\mathrm{TM}}$ Magnetic Particle Processor (Thermo Fisher Scientific) using (1:1:1) pooled libraries (AvLib-1-3). As a positive control, the AvLib-3 library for steroids ${ }^{22}$ was spiked with $100 x$ excess of phages carrying a previously selected sbAvd-2 clone ${ }^{22}$ and panned only against testosterone. For the first round of biopanning, the precipitated phages in PBS were diluted 1:1 with PBST (PBS, $0.05 \%$ Tween-20). The phage dilution (400 $\mu$ l total volume) was combined with $20 \mu \mathrm{g}$ of alkaline phosphatase (AP, for depletion) and $5 \mu$ of the blocked coated magnetic beads and incubated at $4^{\circ} \mathrm{C}$ on a shaker $(500 \mathrm{rpm})$ overnight (eight incubation reactions in parallel containing the control). The magnetic beads used were coated with AP-conjugated progesterone, hydrocortisone, testosterone, cholic acid, ibuprofen, ketoprofen or folic acid. The KingFisher Magnetic Particle Processor was then used to wash the beads six times with $200 \mu \mathrm{l}$ of PBST for $20 \mathrm{~s}$, followed by elution for 20 min with acidic treatment $(0.1 \mathrm{M}$ glycine- $\mathrm{HCl}, \mathrm{pH} 2.2)$. The eluent containing the phages was directly neutralized with $1 \mathrm{M}$ Tris buffer and used to infect freshly grown XL1-Blue cells. Phage amplification was then performed on microplates as described below. Subsequent panning rounds were performed in a similar manner, but the phage culture supernatant was directly used without dilutions, and also the incubations were performed using the KingFisher ${ }^{\mathrm{TM}}$ Magnetic Particle Processor (Thermo Fisher Scientific). The coated magnetic beads were incubated with the phages, collected and re-suspended every 15 min to avoid precipitation at the bottom of the well. The conditions of each of the panning rounds are listed in Supplementary Table 2. Phages were amplified on microplates 
essentially as previously described ${ }^{32}$ with slight modifications: For co-infection, $10 \mu$ VSC-M13 helper phages (Stratagene, La Jolla, CA, USA; $10^{11} \mathrm{pfu} \mathrm{ml}^{-1}$ ) were used. Additionally, during panning rounds $1-$ 3, wt avidin (Fluka, Sigma-Aldrich) was also added to a final concentration of $0.5 \mathrm{mg} \mathrm{ml}^{-1}$ in order to bind the free biotin in the culture medium. Finally, kanamycin $(70 \mu \mathrm{g})$ was added, the temperature was reduced to $28^{\circ} \mathrm{C}$ and the cultivation was continued overnight. Cells were then centrifuged at $16,000 \mathrm{~g}$, for $2 \mathrm{~min}$ at RT. Subsequently, $200 \mu \mathrm{l}$ of the overnight culture supernatant was used for subsequent rounds of panning. The effectiveness of the selection procedure was monitored using the output/input ratios of the phage titers (Supplementary Table 3).

DNA sequencing analysis of the master plates. The protein microplate assay adapted from ${ }^{32}$ was used prior to DNA sequencing to ensure specific ligand binding (not shown). To release the expressed protein from the periplasm, the bacterial cells were lysed with the EasyLyse reagent (Epicentre Biotechnologies) according to the instructions of the manufacturer, and the cell lysate was then used for the microplate assay. For plasmid purification, overnight cultures inoculated from the master plates were used. The plasmids were isolated by automated plasmid purification (using a NucleoSpin Robot-96 Plasmid Kit (Macherey-Nagel) and a pipetting robot (Genesis RSP 100, Tecan)) ${ }^{41}$. The purified plasmids were DNA sequenced as described ${ }^{41}$. DNA sequencing results from the master plate minipreps were translated in the six reading frames, and the correct translation was searched through fuzzy matching to a reference sequence (wt avidin with PelB signal sequence and part of the plll c-terminus), using a Perl script with the String::Approx extension. The translations were selected using a maximum of $30 \%$ identity 
difference criterion. The resulting protein sequences were aligned using the ClustalW alignment algorithm (EBI web server) and further analysed using GeneDoc (v. 2.7.0) ${ }^{48}$ and MEGA $5{ }^{49}$ software.

Subcloning for protein production. The avidin mutants were subcloned into the pET101/D-TOPO ${ }^{\circledR}$ vector ${ }^{22}$ according to the manufacturer's instructions (Invitrogen, Carslbad, CA, USA) with the OmpA signal peptide from Bordetella avium in order to obtain efficient bacterial secretion ${ }^{11}$ and with Cterminal 6x His-tag for purification. The primers Chim_to_1_5', Chim_to_2_5' and Avi_phage_histag_stop_3' (Supplementary Table 4) were used. The subcloning was verified by sequencing.

The point mutation I117Y or N118D was introduced into sbAvd-2 the pET101/D-TOPO ${ }^{\circledR}$ vector by utilizing the QuikChange method using primers I117Y_1 and I117Y_2 or N118D_1 and N118D_2 (Supplementary Table 4) according to the instructions of the manufacturer (Invitrogen).

Protein production. The pET101/D vector based expression constructs were expressed in E. coli BL21(Al) cells (Invitrogen). The fresh transformants were cultured in Lysogeny broth (LB) medium with $0.1 \%(\mathrm{w} / \mathrm{v})$ glucose and ampicillin $\left(100 \mu \mathrm{g} \mathrm{ml}^{-1}\right)$ at $28^{\circ} \mathrm{C}$ shaker. When the culture reached $\mathrm{OD}_{600} 0.4$, the protein expression was induced by adding $0.2 \%(\mathrm{w} / \mathrm{v})$ L-arabinose and IPTG (1mM). The cultivation was continued at $28^{\circ} \mathrm{C}$ for an additional 18 hours before the cells were collected by centrifugation $\left(5000 \mathrm{~g}, 10 \mathrm{~min}, 4^{\circ} \mathrm{C}\right)$ and frozen.

Protein purification. The proteins were purified using affinity chromatography on a Ni-NTA (QIAGEN) column ${ }^{22}$ or with an automated ÄKTA Purifier protein purification system (GE Healthcare Life Sciences) with HisTrap FF Crude columns (GE Healthcare). Bacterial cell pellets were suspended in buffer (20mM NaPO4, $1 \mathrm{M} \mathrm{NaCl}, 20 \mathrm{mM}$ imidazole, $\mathrm{pH} 7.4$ ) and lysed by sonication (Sonics \& Materials 
Vibra Cell ${ }^{\mathrm{TM}}$ ) of lysozyme-treated cells or by homogenization (EmulsiFlex-C3 homogenizator, Avestin Inc.). When sonication was used, $50 \mu \mathrm{g} \mathrm{ml}^{-1}$ lysozyme was added to the cell suspension and incubated on ice for $30 \mathrm{~min}$.

After pooling the elution fractions together, the imidazole concentration was reduced to $20 \mathrm{mM}$ using step-wise dialysis, and the proteins were dialyzed into the measurement buffer $(20 \mathrm{mM}$

$\mathrm{NaH}_{2} \mathrm{PO}_{4} / \mathrm{Na}_{2} \mathrm{HPO}_{4}, 1 \mathrm{M} \mathrm{NaCl}, 20 \mathrm{mM}$ imidazole, $\mathrm{pH}$ 7.4). The purity and quality of the isolated proteins was analyzed by UV/Vis spectrophotometry, SDS-PAGE (followed by Western blotting with polyclonal anti-avidin (University of Oulu, Finland)), and dynamic light scattering (data not shown).

Differential scanning calorimetry (DSC). The thermal stability of the studied proteins in the presence and absence of ligands was analyzed using an automated VP-Capillary DSC System (Microcal Inc.) essentially as described ${ }^{42}$. Thermograms were recorded between 20 and $140^{\circ} \mathrm{C}$ with a heating rate of $2.0^{\circ} \mathrm{C} \mathrm{min}^{-1}$. Protein samples in the measurement buffer were degassed prior to the measurement. The protein concentration in the cell was $7 \mu \mathrm{M}$, and the ligand concentration was $21 \mu \mathrm{M}$, except in the case of fabAvd-1(I117Y) (the protein concentration $14 \mu \mathrm{M}$ and the ligand concentration $42 \mu \mathrm{M}$ ). The results were analyzed using the Origin 7.0 DSC software suite (Microcal Inc.) with multiple non-two-state equations (and Levenberg-Marquardt iterations) for fitting.

Fluorometric assay for ligand binding affinity determination. The measurements were mostly performed using $100 \mathrm{nM}$ protein samples. However, for biotin-binding measurements, up to $350 \mathrm{nM}$ protein samples were used in order to achieve a reasonable total fluorescence quenching due to addition of the ligand. The ligand was added to the protein sample in small aliquots. For proteinligand pairs with a $K_{d}$ value less than $1 \mu \mathrm{M}$, the experiment (originally carried out with 66-30 $000 \mathrm{nM}$ 
ligand concentrations) was repeated using smaller aliquots of the ligand (typically 6-50 $000 \mathrm{nM}$ ) added to the protein sample. The dissociation constant $(\mathrm{Kd})$ was determined from the resulting quenching curve using GraphPad Prism (GraphPad Software, Inc.). The data were fitted to a quadratic equation rearranged from combining two equations: $Y=\frac{B \max \times[\text { free ligand }]}{\mathrm{Kd}+[\text { free ligand }]}+N S \times[$ free ligand $]$ (equation 1) and [free ligand $]=X-Y$ (equation 2). Bmax equals to the total binding capacity (=total protein concentration in the measurement), $\mathrm{X}$ equals to the total concentration of the added ligand, and $N S=\frac{(\mathrm{Ymax}-\mathrm{Ymin})}{(\mathrm{Xmax}-\mathrm{Xmin})}$. The quadratic equation ${ }^{43}$ used for fitting the data: $Y=\mathrm{k} \times$ $\frac{-b+\sqrt{b^{2}-4 a c}}{2 a}$ (equation 3), where $a=-1-N S, b=X(2 \times N S+1)+K d(N S+1)+B \max$, and $c=-X(N S(K d+X)+B \max )$.

In few cases, the last measurement points were excluded resulting in a better fit (increase in $R^{2}$ ).

MD simulations of antidin sbAvd-1 with testosterone A model of sbAvd-1 was prepared using the avidin structure [PDB:1VYO ${ }^{12}$ as a template. Testosterone was docked into the biotin-binding pocket using GOLD ${ }^{44}$. As the testosterone used in biopanning is AP-conjugated via the 3-keto oxygen, we only examined a binding orientation where testosterone enters the binding pocket with its $17-\mathrm{OH}$ group ahead.

Tetrameric sbAvd-1 with four bound testosterone molecules was solvated in TIP3P water, and the system charge was neutralized with chloride ions, resulting in a total of 52,833 atoms. The Amber ff99SB force field ${ }^{50}$ was used for the protein and the GAFF force field ${ }^{51}$ for testosterone, and the simulation was run in NAMD $2.9^{45}$ using a time step of $1 \mathrm{fs}$. Prior to the molecular dynamics simulation, the system was energy minimized in 4 stages using 5000 steps of conjugate gradient 
minimization followed by 50 ps of MD simulation: 1) protein and ligands fixed, 2) C $\alpha$ atoms and ligands fixed, 3) ligand heavy atoms fixed, and 4) all atoms free to move. A 50-ns MD simulation was produced at $300 \mathrm{~K}$ and atmospheric pressure using the Langevin temperature and pressure controls. Analyses and figures were prepared using VMD 1.9.1 52 and Pymol 1.7 (Schrödinger, LLC).

Crystallization and structure determination of sbAvd-2(117Y). The vapor diffusion method was used to crystallize the protein sbAvd-2(I117Y) (1.8 $\mathrm{mg} \mathrm{ml}^{-1}$; in the measurement buffer). Sitting drops having $100 \mathrm{nl}$ of both protein and well solution were prepared with the mosquito ${ }^{\circledR}$ liquid handling robot (TTP Labtech). The well solution derived from the commercial JCSG-plus ${ }^{\mathrm{TM}}$ crystallization screen (NeXtal Tubes Suites, Qiagen) contained 0.18 M sodium chloride, 0.09 M sodium cacodylate (pH 6.5) and 1.8 $\mathrm{M}$ ammonium sulfate. The crystal used for data collection formed within a few weeks on 96well triple sitting drop iQ plates (TTP Labtech) at $21^{\circ} \mathrm{C}$ in a temperature controlled crystallization incubator (RUMED ${ }^{\circledR}$ model 3201). The initial X-ray diffraction properties were analyzed using a PX Scanner (Agilent Technologies). For data collection, $1 \mu \mathrm{L}$ of cryoprotectant $(12.5 \%(\mathrm{v} / \mathrm{v})$ di-ethylene glycol, $12.5 \%$ (v/v) MPD, 37.5\% (v/v) 1,2-propanediol, 12.5\% (v/v) DMSO; CryoProtX'M, Molecular Dimensions) was added to the crystallization drop just prior to freezing in liquid nitrogen. Data were collected at the European Synchrotron Radiation Facility, beam line ID23-2, Grenoble, France (Table 4) and processed using XDS ${ }^{46}$. Initial phase estimates for the structure factors were obtained using the molecular replacement program Phaser ${ }^{53}$ within the CCP4i GUI ${ }^{54,55}$. The Matthews coefficient ${ }^{56}$ predicted two molecules per asymmetric unit, but for molecular replacement, a monomer of AVR2 [PDB:1WBI] ${ }^{57}$ was used as the search model; the space group was confirmed to be $\mathrm{P} 2{ }_{1} 2{ }_{1} 2$ after the replacement. Refmac5 ${ }^{47}$ was used for refinement, and the structure was rebuilt in several cycles and manually edited using $\operatorname{Coot}^{58}$ (see see Table 4, for refinement statistics). Solvent atoms were added 
using ARP/wARP 59-62 and in Coot. PyMOL (The PyMOL Molecular Graphics System, Version 1.5.0.4 Schrödinger, LLC.) and Bodil ${ }^{63}$ were used to check the final structure and for creating figures. The final structure was validated using the inbuilt tools of $\operatorname{Coot}^{58}$, and by using MolProbity ${ }^{64}$ within the Phenix software suite ${ }^{65}$. The final coordinates and structure factors of sbAvd-2(I117Y) were deposited in the Protein Data Bank ${ }^{66,67}$ with the accession code $4 \mathrm{U} 46$. 


\section{Supplemental References}

(48) Nicholas, K.,B., Nicholas, Hugh, B.,Jr., and Deerfield, D.,W. II. (1997) GeneDoc: Analysis and Visualization of Genetic Variation. EMBnet.news 4, 1-4.

(49) Tamura, K., Peterson, D., Peterson, N., Stecher, G., Nei, M., and Kumar, S. (2011) MEGA5: molecular evolutionary genetics analysis using maximum likelihood, evolutionary distance, and maximum parsimony methods. Mol. Biol. Evol. 28, 2731-2739.

(50) Jones, G., Willett, P., Glen, R. C., Leach, A. R., and Taylor, R. (1997) Development and validation of a genetic algorithm for flexible docking. J. Mol. Biol. 267, 727-748.

(51) Wang, J., Wolf, R. M., Caldwell, J. W., Kollman, P. A., and Case, D. A. (2004) Development and testing of a general amber force field. J. Comput. Chem. 25, 1157-1174.

(52) Humphrey, W., Dalke, A., and Schulten, K. (1996) VMD: visual molecular dynamics. J. Mol. Graph. 14, 33-8, 27-8.

(53) McCoy, A. J., Grosse-Kunstleve, R. W., Adams, P. D., Winn, M. D., Storoni, L. C., and Read, R. J. (2007) Phaser crystallographic software. J. Appl. Crystallogr. 40, 658-674.

(54) Collaborative Computational Project, Number 4 (1994) The CCP4 suite: programs for protein crystallography. Acta Crystallogr. D Biol. Crystallogr. 50, 760-763.

(55) Potterton, E., Briggs, P., Turkenburg, M., and Dodson, E. (2003) A graphical user interface to the \it CCP4 program suite. Acta Crystallographica Section D 59, 1131-1137.

(56) Matthews, B. W. (1968) Solvent content of protein crystals. J. Mol. Biol. 33, 491-497.

(57) Hytonen, V. P., Maatta, J. A., Kidron, H., Halling, K. K., Horha, J., Kulomaa, T., Nyholm, T. K., Johnson, M. S., Salminen, T. A., Kulomaa, M. S., and Airenne, T. T. (2005) Avidin related protein 2 shows unique structural and functional features among the avidin protein family. BMC Biotechnol. 5, 28.

(58) Emsley, P., Cowtan, K. (2004) Coot: model-building tools for molecular graphics. Acta Crystallogr. D Biol. Crystallogr. 60, 2126-2132.

(59) Lamzin, V. S., Wilson, K. S. (1993) Automated refinement of protein models. Acta Crystallogr. D Biol. Crystallogr. 49, 129-147.

(60) Langer, G., Cohen, S. X., Lamzin, V. S., and Perrakis, A. (2008) Automated macromolecular model building for X-ray crystallography using ARP/wARP version 7. Nat. Protoc. 3, 1171-1179.

(61) Morris, R. J., Perrakis, A., and Lamzin, V. S. (2003) ARP/wARP and automatic interpretation of protein electron density maps. Methods Enzymol. 374, 229-244. 
(62) Perrakis, A., Morris, R., and Lamzin, V. S. (1999) Automated protein model building combined with iterative structure refinement. Nat. Struct. Biol. 6, 458-463.

(63) Lehtonen, J., Still, D., Rantanen, V., Ekholm, J., Bjorklund, D., Iftikhar, Z., Huhtala, M., Repo, S., Jussila, A., Jaakkola, J., Pentikainen, O., Nyronen, T., Salminen, T., Gyllenberg, M., and Johnson, M. (2004) BODIL: a molecular modeling environment for structure-function analysis and drug design. J. Comput. Aided Mol. Des. 18, 401-419.

(64) Davis, I. W., Leaver-Fay, A., Chen, V. B., Block, J. N., Kapral, G. J., Wang, X., Murray, L. W., Arendall, W. B.,3rd, Snoeyink, J., Richardson, J. S., and Richardson, D. C. (2007) MolProbity: all-atom contacts and structure validation for proteins and nucleic acids. Nucleic Acids Res. 35, W375-83.

(65) Adams, P. D., Grosse-Kunstleve, R. W., Hung, L. W., loerger, T. R., McCoy, A. J., Moriarty, N. W., Read, R. J., Sacchettini, J. C., Sauter, N. K., and Terwilliger, T. C. (2002) PHENIX: building new software for automated crystallographic structure determination. Acta Crystallogr. D Biol. Crystallogr. 58, 1948-1954.

(66) Berman, H. M., Battistuz, T., Bhat, T. N., Bluhm, W. F., Bourne, P. E., Burkhardt, K., Feng, Z., Gilliland, G. L., Iype, L., Jain, S., Fagan, P., Marvin, J., Padilla, D., Ravichandran, V., Schneider, B., Thanki, N., Weissig, H., Westbrook, J. D., and Zardecki, C. (2002) The Protein Data Bank. Acta Crystallogr. D Biol. Crystallogr. 58, 899907.

(67) Berman, H. M., Westbrook, J., Feng, Z., Gilliland, G., Bhat, T. N., Weissig, H., Shindyalov, I. N., and Bourne, P. E. (2000) The Protein Data Bank. Nucleic Acids Res. 28, 235-242. 